\title{
Gelation of Aqueous Pectin Solutions: A Dynamic Light Scattering Study
}

\author{
Janaky Narayanan, ${ }^{*}, 1$ Vinod W. Deotare, ${ }^{*}$ Ranjini Bandyopadhyay, $\dagger$ and A. K. Sood $\dagger$ \\ * Department of Physics, R. J. College, Ghatkopar (W), Mumbai 400 086, India; and $\dagger$ Department of Physics, \\ Indian Institute of Science, Bangalore 560 012, India
}

Received May 2, 2001; accepted October 22, 2001

\begin{abstract}
We report the dynamic light scattering study of the gelation of aqueous solutions of the biopolymer, pectin, induced by the addition of calcium chloride. The time correlation function data are analyzed under the framework of the coupling model. As the solution enters the semidilute regime where gelation sets in, the relaxation process shows a stretched exponential behavior. The stretching exponent decreases and the characteristic time of the stretched exponential diverges as the system evolves to a gel. Aqueous pectin solutions in the presence of $0.1 \mathrm{M} \mathrm{NaCl}$ show similar behavior. Thus, the molecular relaxation modes of pectin solutions can be well described by the coupling model. (c) 2002 Elsevier Science

Key Words: pectin; dynamic light scattering; coupling model; sol-gel transition.
\end{abstract}

\section{INTRODUCTION}

Polymer species can form a variety of supramolecular structures via gelation, aggregation, phase separation, and ordering. Gels can be prepared by chemically cross-linking branched polymer chains or physical aggregation of polymer chains into threedimensional structure (1). Hydrogen bonding, Coulomb interaction, and dipolar forces are some of the types of bonding which lead to network formation resulting in gelation. It is sometimes possible to break these types of bonds by increasing the temperature. Systems with these properties are said to form thermoreversible gels.

Dynamics and relaxation process in sol-gel systems have been the subject of continued interest (2-7). In the semidilute solutions of polymers and polymer-related systems, topological constraints on the polymer molecular motion due to polymer overlap strongly affect the dynamics. As gelation progresses through the semidilute solution, the complexity of the phenomenon increases. Dynamic light scattering (DLS) has served as a useful technique to study the dynamics of diffusion of polymers in solutions. In this paper we present a DLS study of gelation in aqueous solutions of the biopolymer, pectin. The decay rate of the time correlation function of the scattered electric field is determined as the system undergoes sol-gel transition and the

\footnotetext{
${ }^{1}$ To whom correspondence should be addressed. Fax: +91-22-522 4413. E-mail: janaky@vsnl.com.
}

results are analyzed in the framework of the coupling model, devised by Ngai and co-workers (8-13).

Pectins are important polysaccharides, which are used in the food industry as gelling agents (14). Apple pomace and orange peels are the two major sources of commercial pectins. In native pectins, about $70 \%$ of the carboxyl groups are methylesterified. These "high methoxyl" (HM) pectins form gels in acidic medium in the presence of high concentrations of sucrose. Pectins of lower ester content are produced by controlled deesterification of native pectins and the resulting polysaccharide forms gels upon addition of calcium or related divalent ions (15). In these "low-methoxyl" (LM) pectins, gelation results from ionic linkages via $\mathrm{Ca}^{2+}$ ion bridges between two carboxyl groups belonging to two different chains (16). Gelation of LM pectins is affected by several intrinsic and extrinsic parameters such as the degree of methylation, the average molecular weight of the sample, the ionic strength, the $\mathrm{pH}$, the temperature. An increase in the ionic strength, a neutral $\mathrm{pH}$, and a decrease in the setting temperature and in the degree of methylation lower the amount of calcium chloride required to obtain the sol-gel transition (17). Molecular weight distribution and conformation of high methoxy citrus pectins were studied using light scattering and gel-permeation chromatography with low-speed sedimentation equilibrium $(18,19)$. Solution conformation of apple and citrus pectins with a large range of degree of methylation was studied by small angle neutron scattering, viscometry, and molecular modeling (20). These studies showed that pectins are relatively stiff molecules with persistence length and flexibility of the polymeric backbone dependent on the degree of methylation. In a good solvent such as $0.1 \mathrm{M} \mathrm{NaCl}$ aqueous solution at $25^{\circ} \mathrm{C}$, the pectin molecules exhibit the general behavior of linear flexible polymers in good solvent (21). The sol-gel transition brought forth by increasing calcium chloride concentration was monitored by rheological measurements close to the gel point (22). The dynamic exponents for viscosity and elastic moduli were found to be consistent with the predictions of the scalar elasticity percolation model (23-25). Thus in the evolution from sol to gel, the pectin solution can be expected to exhibit molecular relaxation modes characteristic of semidilute polymer solutions.

Dilute solutions of monodisperse polymers as well as some semidilute solutions of flexible polymers at good solvent 
conditions show single exponential relaxation behavior of the concentration fluctuations that can be probed by DLS experiments. However, for several associating polymer solutions (26), polymeric cluster solutions (2), and thermoreversible gelling solutions (3-7), a broad distribution of relaxation times is often recorded. For these systems, DLS showed an initial exponential decay followed by a stretched exponential with width parameter, $\beta$. The characteristic time of the stretched exponential diverged to infinity as the system set to a gel. In some cases such as gelatine sol-gel transition $(5,7)$, intermediate power law decay was also observed. The coupling model of Ngai and co-workers is found to be well adapted for interpreting the experimental findings in several complex systems exhibiting a stretched exponential mode of relaxation (8). In this DLS study on aqueous solutions of pectin, we find that most of the experimental findings are in accordance with the coupling model. Though several aspects of sol-gel transition in aqueous pectin solutions have been thoroughly investigated by Axelos and co-workers (17, 21, $22,27)$, to the best of our knowledge, this is the first DLS study which applies coupling model to the sol-gel transition of pectin solutions. The present study strengthens the findings of Axelos and co-workers on gelation of pectin solutions.

\section{EXPERIMENTAL DETAILS}

Pectin from citrus fruit (Potassium salt) with degree of esterification 22\% was obtained from Sigma Chemicals (P-9311) and was used without further purification. The manufacturer has quoted the viscosity average molecular weight of this product to be 17,000 . Calcium chloride dihydrate $\left(\mathrm{CaCl}_{2} \cdot 2 \mathrm{H}_{2} \mathrm{O}\right)$ and sodium chloride $(\mathrm{NaCl})$ were bought from Merck and used as received. Stock solutions of calcium chloride and sodium chloride of appropriate concentrations were prepared. Pectin concentrations of 2.5 and $10.0 \mathrm{~g} \mathrm{~L}^{-1}$ were chosen for DLS studies. Homogeneous samples were obtained by mixing aqueous pectin solution with calcium chloride and sodium chloride solutions of appropriate volumes to get the required molarity of the salts. The solution was stirred for half an hour and then poured into 1-cmdiameter glass vial for DLS studies. The measurements were made after the sample was cured for $24 \mathrm{~h}$ at room temperature.

The DLS measurements on $2.5 \mathrm{~g} \mathrm{~L}^{-1}$ pectin solutions were made at the Indian Institute of Science, Bangalore, using a homemade photon correlation spectrometer employing $\mathrm{Kr}^{+}$laser $(\lambda=647 \mathrm{~nm})$ and Malvern digital correlator at 60 , 90 , and $120^{\circ}$ scattering angles. Measurements on $10 \mathrm{~g} \mathrm{~L}^{-1}$ pectin solutions were made at Novel Materials and Structural Chemistry Division, Bhabha Atomic Research Center, Mumbai, using a standard Brook Haven light scattering instrument BI-90 with $5 \mathrm{~mW}$ He-Ne Laser $(\lambda=632.8 \mathrm{~nm})$, the angle of scattering being $90^{\circ}$. In DLS experiments, the normalized intensity autocorrelation function of the scattered light, $g^{(2)}(q, t)=\langle I(q, t) I(q, 0)\rangle /\langle I\rangle^{2}$ is measured and is used to calculate the normalized time correlation function of the scattered electric field, $g^{(1)}(q, t)=\left\langle E^{*}(q, t) E(q, 0)\right\rangle /\langle E\rangle^{2}$. The function $g^{(1)}(q, t)$ can be identified with the dynamic structure factor $S(q, t)$ which, for a Gaussian signal, is related to $g^{(2)}(q, t)$ by the Seigert relation $g^{(2)}(q, t)=1+f|S(q, t)|^{2}$, where the coherence factor $f$ measures the degree of coherence of the scattered light (28). Four or more different sampling times were used to obtain the scattered intensity autocorrelation function $\langle I(0) I(t)\rangle$ and then the data were spliced together. The background subtracted normalized dynamic structure factor

$$
S(q, t)=\sqrt{\frac{g^{(2)}(t)-1}{f}}
$$

was calculated from the intensity autocorrelation function. $f$ is determined from the value of the intercept of the plot of $g^{(2)}(q, t)$ vs $t$. Here, $q$ is the scattering wave vector given by

$$
q=\frac{4 \pi \mu \sin (\theta / 2)}{\lambda}
$$

$\mu$ being the refractive index of the solution and $\theta$, the scattering angle. The data were fitted using (26)

$$
S(q, t)=A_{f} \exp \left(-t / \tau_{f}\right)+A_{s} \exp \left(-\left(t / \tau_{s c}\right)^{\beta}\right),
$$

where $A_{f}$ and $A_{s}$ are the amplitudes of the fast and slow relaxation modes, respectively, with $A_{f}+A_{s}=1 . \tau_{f}$ and $\tau_{s}$ are the characteristic times of fast and slow decays with

$$
\tau_{s}=\frac{\tau_{s c}}{\beta} \Gamma(1 / \beta),
$$

where $\beta$, the stretching exponent, is a measure of the width of the distribution of relaxation times and $\Gamma(1 / \beta)$ is the gamma function.

The viscosity measurements were carried out with the use of Ubbelohde viscometer on pectin solutions in the concentration range 0.1 to $20 \mathrm{~g} \mathrm{~L}^{-1}$ in order to determine $C^{*}$, the crossover concentration from the dilute to the semidilute regime of the pectin solutions.

\section{THEORETICAL CONSIDERATIONS}

The coupling model (8-13) is a general approach to dynamics of the constrained or interacting systems. According to this model, there exists a temperature-insensitive crossover time, $t_{C}$ such that for $t<t_{C}$, the relaxation process is not affected by coupling effects. The basic units relax independently with a single exponential correlation function. At long times, i.e., $t>t_{C}$, dynamic constraints come into play and give rise to fractional decay of the correlation function. Thus,

$$
\phi(t)=\exp \left(-t / \tau_{0}\right) \quad \text { for } t<t_{C}
$$


and

$$
\phi(t)=\exp \left(-\left(\frac{t}{\tau}\right)^{1-n}\right) \quad \text { for } t>t_{C} .
$$

$\tau_{0}$ is the initial relaxation time in the absence of coupling, $\tau$ is the effective relaxation time, $t_{C}$ is the characteristic time beyond which coupling effects influence the dynamics, and $n$ is the coupling parameter in the range $0 \leq n \leq 1$ and depends on the intermolecular interaction. For continuity of $\phi(t)$ at $t=t_{C}$,

$$
\tau=\left(t_{c}^{-n} \tau_{0}\right)^{1 /(1-n)}
$$

When the condition $\tau_{0} / t_{C} \gg 1$ holds, the linear exponential, namely, $\exp \left(-t / \tau_{0}\right)$ decays by an insignificant amount at $t=t_{C}$ and the correlation function is practically given at all times by the stretched exponential.

The concentration fluctuation correlation function is given by

$$
\phi(t)=\frac{\left\langle\delta c_{q}(t) \delta c_{-q}(0)\right\rangle}{\left\langle\left|\delta c_{q}\right|^{2}\right\rangle}
$$

where $\delta c_{q}(t)$ is the concentration fluctuation of wave vector $q$. The function $\phi(t)$ is identified with $S(q, t)$. Hence $\tau_{0}$ in Eq. [5] is the correlation time of free diffusion given by

$$
\tau_{0}(q)=\left(D_{0} q^{2}\right)^{-1}
$$

where $D_{0}$ is the free translational diffusion coefficient of the center of mass of the polymer. The experimentally observed width parameter $\beta$ in Eq. [3] is related to the coupling parameter $n$ through $\beta=1-n$. Substituting Eq. [9] in Eq. [7] gives

$$
\tau \propto q^{-2 / \beta} .
$$

We recall that the theoretical approach of the coupling model is semiempirical, as it does not identify the particular mechanism or the exact nature of the interaction responsible for the coupling. However, it has been very effective in describing various relaxation processes in complex systems. It has been demonstrated (29) that general results from anomalous diffusion in disordered fractal-like systems produce the features of the time-dependent rate proposed by the coupling model.

\section{RESULTS AND DISCUSSION}

The intrinsic viscosity $[\eta]$ was determined by using Huggin's and Kraemer's extrapolation equations.

Huggin's equation:

$$
\eta_{s p} / C=[\eta]+k_{1}[\eta]^{2} C
$$

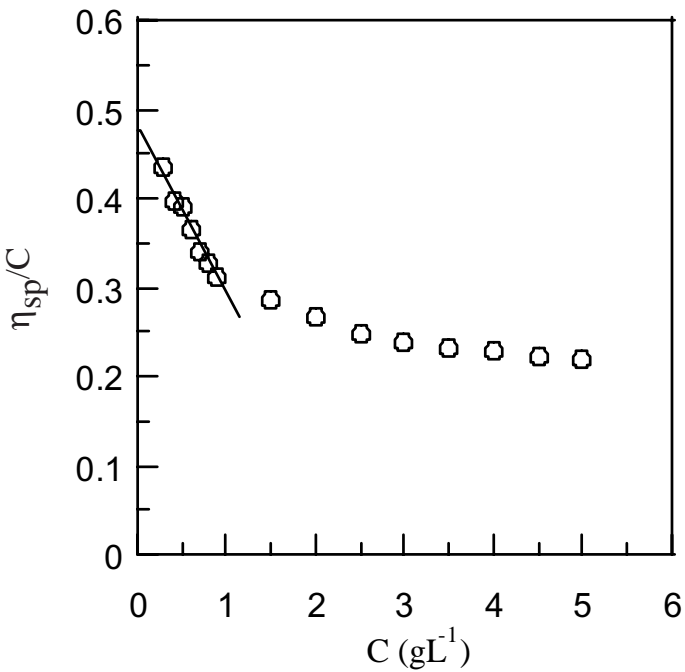

FIG. 1. Plot of $\eta_{s p} / C$ against $C$. The intrinsic viscosity $[\eta]$ given by the intercept is $475 \mathrm{ml} \mathrm{g}^{-1}$. The deviation from linearity occurs at $C^{*} \sim 1.5 \mathrm{~g} \mathrm{~L}^{-1}$.

Kraemer's equation:

$$
\left(\ln \eta_{r}\right) / C=[\eta]+k_{2}[\eta]^{2} C .
$$

$k_{1}$ and $k_{2}$ are the interaction parameters characteristic of the polymer-solvent system, $\eta_{r}=\eta / \eta_{s}$ is the relative viscosity of the solution with respect to the solvent and $\eta_{s p}$ is the specific viscosity given by $\eta_{s p}=\eta_{r}-1$. The concentration range chosen for the double extrapolation was 0.3 to $0.9 \mathrm{~g} \mathrm{~L}^{-1}$ corresponding to $\eta_{r}$ in the range 1.1 to 1.3 . The intrinsic viscosity was found to be $475 \mathrm{ml} \mathrm{g}^{-1}$. Figure 1 shows the plot of $\left(\eta_{s p} / C\right)$ against the concentration of the polymer, $C$. The deviation from linearity as given by Huggin's equation occurs at $C^{*} \sim 1.5 \mathrm{~g} \mathrm{~L}^{-1}$. For a polymer solution (30), the overlap concentration from dilute to semidilute regime is expected to be at $C^{*}=$ $0.77 /[\eta]$. The value of $C^{*} \sim 1.5 \mathrm{~g} \mathrm{~L}^{-1}$ is close to this expected value.

Garnier et al. (17) had determined the phase diagrams of citrus pectins of large range of degree of methoxylation. The different phases (sol, gel, and syneresis) observed in pectin solutions on addition of calcium chloride solution were presented by plotting molar concentration of calcium chloride versus the reduced polymer concentration $C[\eta]$. The sol-gel transition curves showed that the minimal polymer concentration able to promote gelation, $C_{0}$, depended on the solvent conditions. In the presence of an excess of monovalent salt $(0.1 \mathrm{M} \mathrm{NaCl})$, gelation was possible before the overlap threshold concentration, $C^{*}$, and the amount of calcium required to obtain a gel was independent of the polymer concentration for $C>C^{*}$. In salt-free solutions, $C_{0}$ was very near $C^{*}$ and the amount of calcium required to obtain a gel increased with polymer concentration. Guided by these results, we selected two pectin concentrations, 2.5 and $10 \mathrm{~g} \mathrm{~L}^{-1}$ for the DLS study of the sol-gel transition induced by calcium chloride in aqueous salt-free pectin solutions. The gelation in 
$10 \mathrm{~g} \mathrm{~L}^{-1}$ pectin solution in the presence of $0.1 \mathrm{M} \mathrm{NaCl}$ was also studied.

\section{A. Pectin Gelation in the Absence of $\mathrm{NaCl}$}

For a pectin concentration of $2.5 \mathrm{~g} \mathrm{~L}^{-1}$, the sol-gel transition was investigated by increasing calcium chloride concentration from 0 to $3.5 \mathrm{mM}$. After curing for $24 \mathrm{~h}$, if there was flow in the sample when the sample vial was tilted, the system was considered to be sol. If there was no deformation of the meniscus when the vial was tilted, the sample was considered as gel. The samples with 3.0 and $3.5 \mathrm{mM}$ calcium chloride were gels. Above $3.5 \mathrm{mM}$, syneresis was observed with the presence of water above gel surface. For the gelled samples, the DLS data were collected at scattering angles $90^{\circ}\left(q=1.826 \times 10^{7} \mathrm{~m}^{-1}\right)$, while for other samples the data were collected for scattering angles 60,90 , and $120^{\circ}$.

Figure 2 depicts the dynamic structure factor $S(q, t)$ obtained for $2.5 \mathrm{~g} \mathrm{~L}^{-1}$ aqueous pectin solutions in the presence of 0 , 2.5 , and $3.5 \mathrm{mM}$ calcium chloride at scattering angle $90^{\circ}$. The theoretical fits to the data using Eq. [3] are also shown. As the concentration of calcium chloride increases there is a progressive slowing down of the relaxation process. This type of slowing down has been observed in a number of DLS studies $(2-7,26)$. Figures 3 and 4 show the variation of the amplitudes of the fast and slow relaxation modes with calcium chloride concentration. With increase in calcium chloride concentration, the coupling effects influence the relaxation process as the solution is driven from sol to gel phase. Hence the slow mode becomes more dominant at higher calcium concentrations.

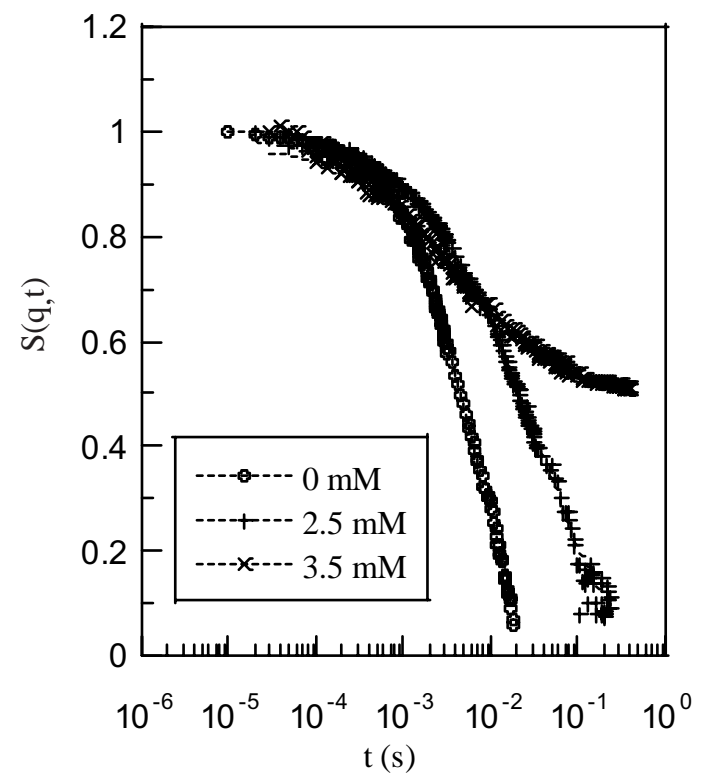

FIG. 2. The dynamic structure factor $S(q, t)$ for $2.5 \mathrm{~g} \mathrm{~L}^{-1}$ aqueous pectin solution as a function of time in the presence of $0,2.5$, and $3.5 \mathrm{mM} \mathrm{CaCl}_{2}$. Dotted lines are theoretical fits using Eq. [3].

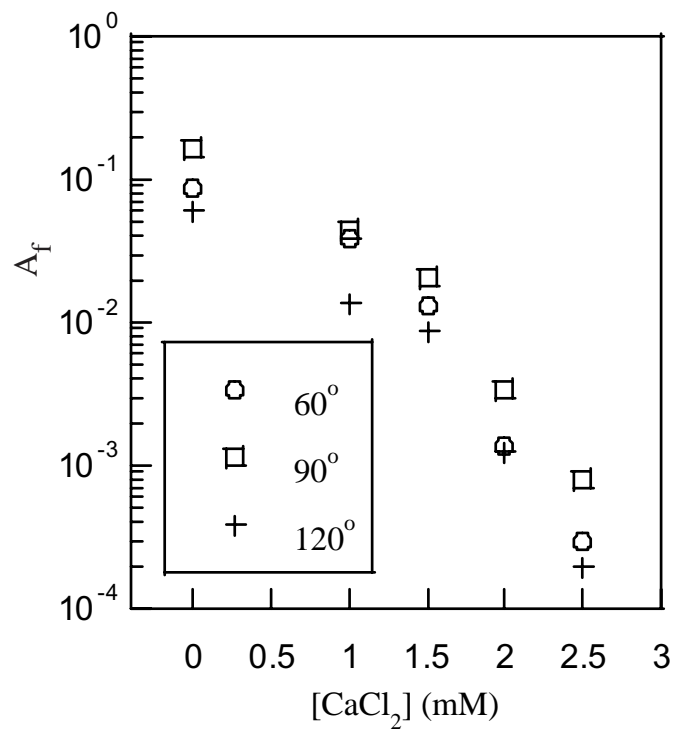

FIG. 3. Variation of the amplitude, $A_{f}$, of the fast relaxation mode of $S(q, t)$ of $2.5 \mathrm{~g} \mathrm{~L}^{-1}$ aqueous pectin solution with $\mathrm{CaCl}_{2}$ concentration for three different $q$ values. (i) $q=1.29 \times 10^{7} \mathrm{~m}^{-1}\left(\theta=60^{\circ}\right)$; (ii) $q=1.83 \times 10^{7} \mathrm{~m}^{-1}\left(\theta=90^{\circ}\right)$; (iii) $q=2.24 \times 10^{7} \mathrm{~m}^{-1}\left(\theta=120^{\circ}\right)$.

The variation of the $q$-independent width exponent, $\beta$, with calcium chloride concentration is shown in Fig. 5. In pure pectin solution the value of $\beta$ is 0.985 and it decreases to 0.22 at $\left[\mathrm{CaCl}_{2}\right]=3.5 \mathrm{mM}$ when the sample becomes a gel. The variations of the fast and slow relaxation times, $\tau_{f}$ and $\tau_{s}$, respectively, at scattering angle $90^{\circ}$ are depicted in Fig. 6 . The slow relaxation time increases by orders of magnitude when sol-gel transition occurs. From Eq. [9], we find that the fast time $\tau_{f}$ is

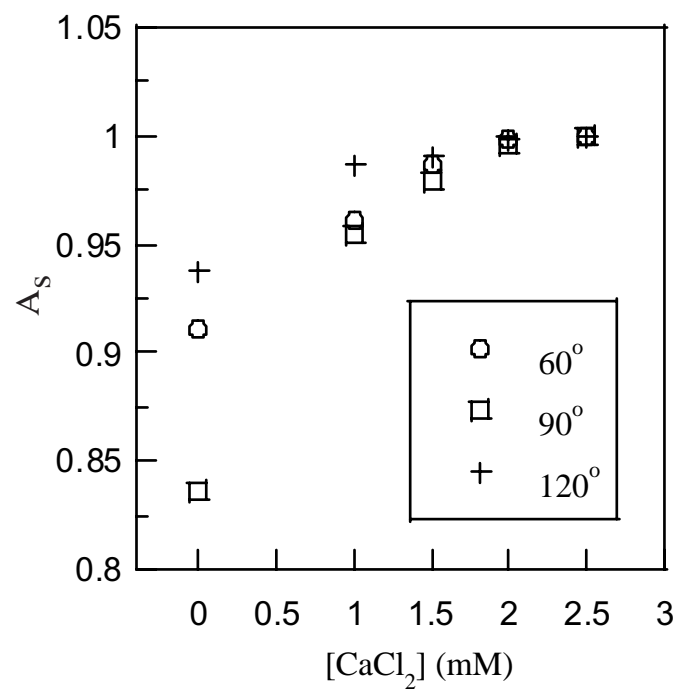

FIG. 4. Variation of the amplitude, $A_{s}$, of the slow relaxation mode of $S(q, t)$ of $2.5 \mathrm{~g} \mathrm{~L}^{-1}$ aqueous pectin solution with $\mathrm{CaCl}_{2}$ concentration for three different $q$ values. (i) $q=1.29 \times 10^{7} \mathrm{~m}^{-1}\left(\theta=60^{\circ}\right)$; (ii) $q=1.83 \times 10^{7} \mathrm{~m}^{-1}$ $\left(\theta=90^{\circ}\right)$; (iii) $q=2.24 \times 10^{7} \mathrm{~m}^{-1}\left(\theta=120^{\circ}\right)$. 


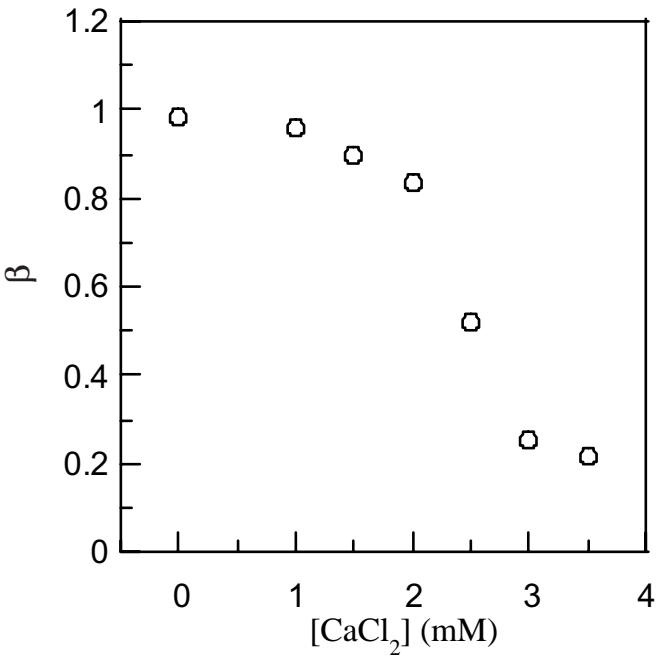

FIG. 5. Variation of the $q$-independent stretching exponent, $\beta$, for $2.5 \mathrm{~g} \mathrm{~L}^{-1}$ aqueous pectin solution with $\mathrm{CaCl}_{2}$ concentration.

related to the diffusion coefficient, $D_{0}$, by $D_{0}=\left(\tau_{f} q^{2}\right)^{-1}$. For aqueous pectin solution in the absence of calcium chloride, for the three $q$ values used in this study, $D_{0} \sim 2.0 \times 10^{-12} \mathrm{~m}^{2} \mathrm{~s}^{-1}$. This slow diffusion process may be due to the reasons that the solution is already in the semidilute regime $\left(C>C^{*}\right)$ and the pectin molecules are stiffer in salt-free solution due to charge effects.

The $q$ dependence of $\tau_{f}$ and $\tau_{s}$ are shown in Figs. 7 and 8 on $\log$-log plots. The coupling model predicts $\tau_{f}$ and $\tau_{s}$ to scale as $\tau_{f} \sim q^{-\alpha_{f}}$ and $\tau_{s} \sim q^{-\alpha_{s}}$, where $\alpha_{f}=2$ for diffusive mode and $\alpha_{s}=2 / \beta$. The $\alpha$ values obtained for the variation of $\tau_{f}$ and $\tau_{s}$ with $q$ are listed in Table 1 . The results by and large conform to the predictions of coupling model.

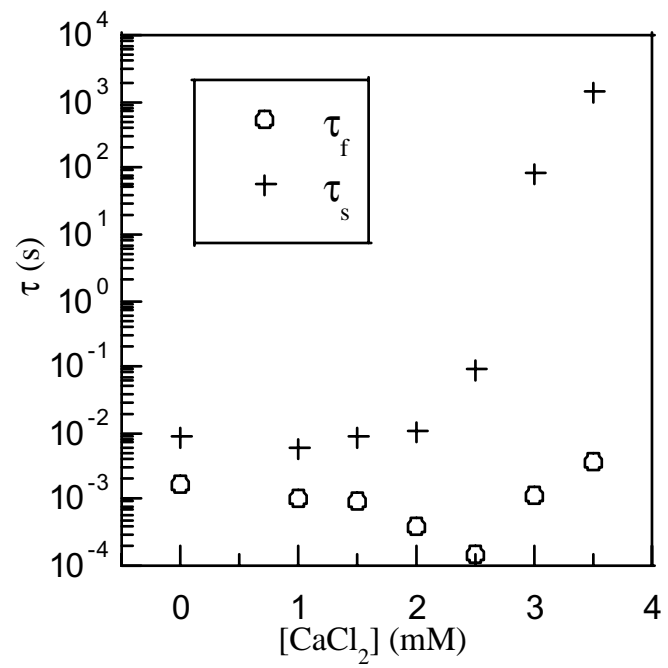

FIG. 6. Variation of the characteristic times $\tau_{f}$ and $\tau_{s}$ of the fast and slow relaxation modes of $S(q, t)$ of $2.5 \mathrm{~g} \mathrm{~L}^{-1}$ aqueous pectin solution with $\mathrm{CaCl}_{2}$ concentration.

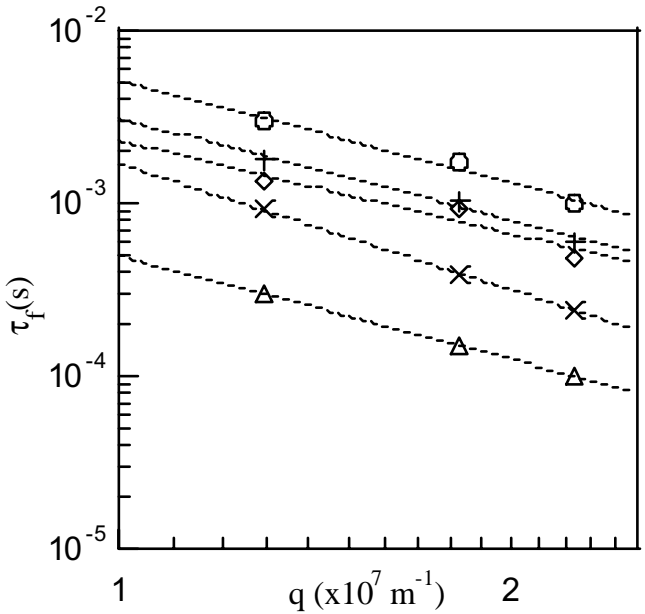

FIG. 7. Variation of $\tau_{f}$ with $q$ for $2.5 \mathrm{~g} \mathrm{~L}^{-1}$ aqueous pectin solution for various $\mathrm{CaCl}_{2}$ concentrations: $0 \mathrm{mM}(\bigcirc) ; 1 \mathrm{mM}(+) ; 1.5 \mathrm{mM}(\diamond) ; 2 \mathrm{mM}(\times)$; $2.5 \mathrm{mM}(\triangle)$.

The gelation of salt-free aqueous pectin solution of $10 \mathrm{~g} \mathrm{~L}^{-1}$ concentration was also studied at scattering angle $90^{\circ}(q=$ $1.872 \times 10^{7} \mathrm{~m}^{-1}$ ) using BI-90 particle sizer employing He-Ne laser. The calcium chloride concentration was varied from 0 to $4.5 \mathrm{mM}$. In this range of calcium chloride concentration, the system showed viscosification, while there was gel formation at $\left[\mathrm{CaCl}_{2}\right] \geq 8 \mathrm{mM}$. This is in accordance with the observation of Garnier et al. (17), that in salt-free pectin solutions the amount of calcium for gel formation increases with the polymer concentration.

Figure 9 shows the variations of the amplitudes and characteristic times of fast and slow relaxation modes and the stretching exponent with calcium chloride concentration for $10 \mathrm{~g} \mathrm{~L}^{-1}$

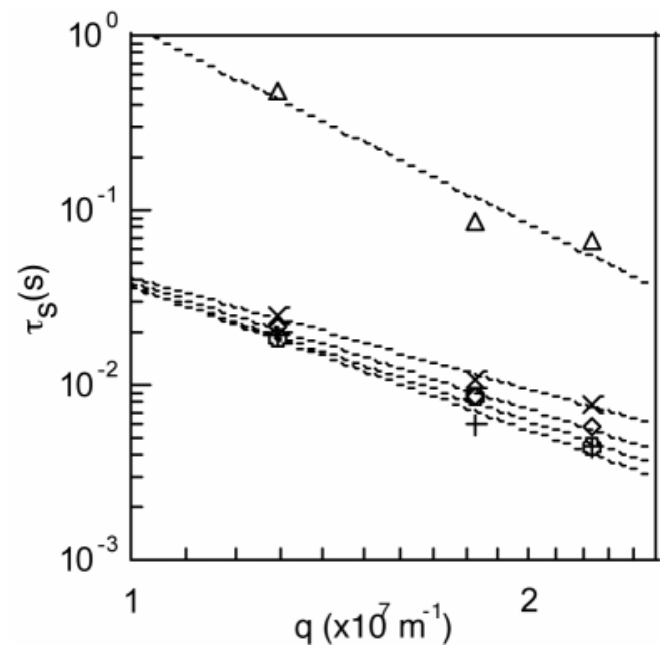

FIG. 8. Variation of $\tau_{s}$ with $q$ for $2.5 \mathrm{~g} \mathrm{~L}^{-1}$ aqueous pectin solution for various $\mathrm{CaCl}_{2}$ concentrations: $0 \mathrm{mM}(\bigcirc) ; 1 \mathrm{mM}(+) ; 1.5 \mathrm{mM}(\diamond) ; 2 \mathrm{mM}(\times)$; $2.5 \mathrm{mM}(\triangle)$. 
TABLE 1

Values of Exponents $\alpha_{f}$ and $\alpha_{s}$ Obtained for the Variation of the Characteristic Times $\tau_{f}$ and $\tau_{s}$, Respectively, with the Wave Vector $q$ and the Values of the Stretching Exponent $\beta$ for $2.5 \mathrm{~g} \mathrm{~L}^{-1}$ Aqueous Pectin Solution in the Presence of Different $\mathrm{CaCl}_{2}$ Concentrations

\begin{tabular}{cccc}
\hline$\left[\mathrm{CaCl}_{2}\right]$ in $\mathrm{mM}$ & $\alpha_{f}$ & $\alpha_{s}$ & \multicolumn{1}{c}{$\beta$} \\
\hline 0 & 1.96 & 2.55 & 0.985 \\
1.0 & 1.95 & 2.74 & 0.956 \\
1.5 & 1.78 & 2.42 & 0.9 \\
2.0 & 2.43 & 2.11 & 0.835 \\
2.5 & 2.00 & 3.77 & 0.52 \\
\hline
\end{tabular}

aqueous pectin solution. The general behavior of these parameters is similar to that found in $2.5 \mathrm{~g} \mathrm{~L}^{-1}$ aqueous pectin solution. However, since the samples are in a pregel state, the parameters do not show the drastic variation observed in samples under-

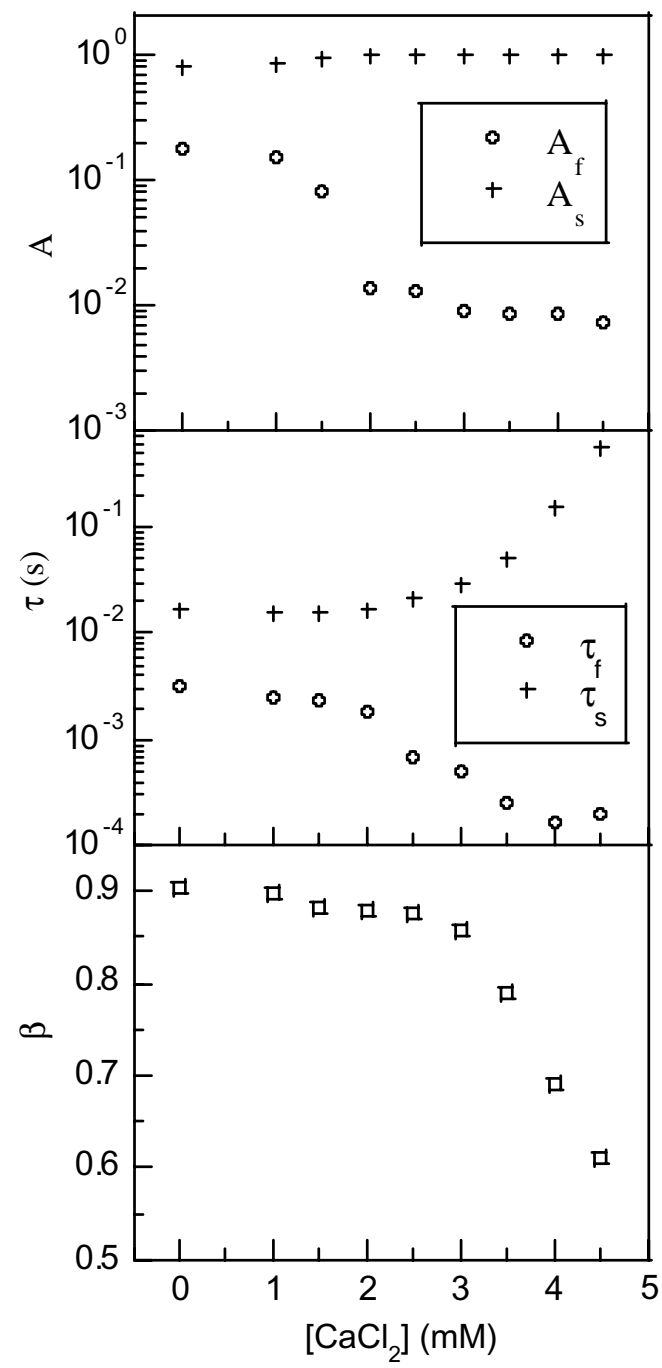

FIG. 9. Variation of amplitudes, characteristic times, and stretching exponent of the relaxation modes of $S(q, t)$ for $10 \mathrm{~g} \mathrm{~L}^{-1}$ pectin solution with $\mathrm{CaCl}_{2}$ concentration.

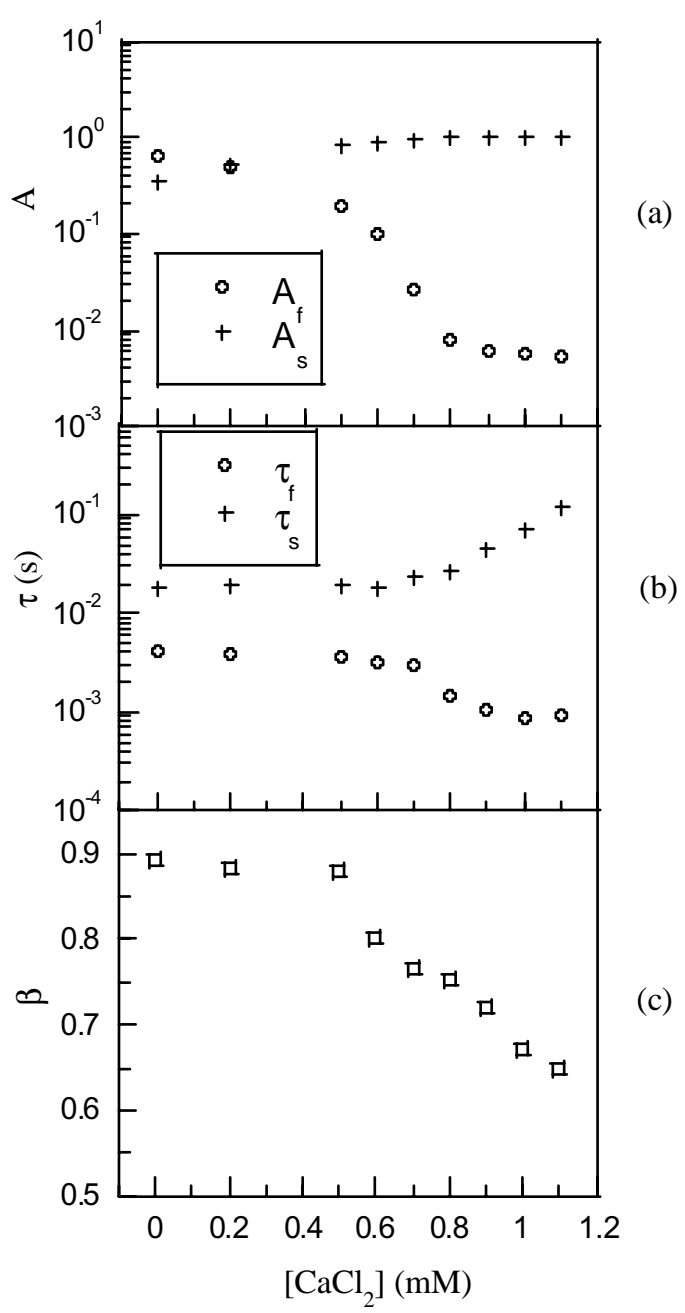

FIG. 10. Variation of amplitudes, characteristic times, and stretching exponent of the relaxation modes of $S(q, t)$ for $10 \mathrm{~g} \mathrm{~L}^{-1}$ pectin solution in the presence of $0.1 \mathrm{M} \mathrm{NaCl}$ with $\mathrm{CaCl}_{2}$ concentration.

going sol-gel transition. The width parameter, $\beta$, for salt-free $10 \mathrm{~g} \mathrm{~L}^{-1}$ pectin solution is 0.9 which shows that the coupling effects are quite strong since the solution is close to the concentrated regime.

\section{B. Pectin Gelation in the Presence of $\mathrm{NaCl}$}

Figure 10 shows the variations of the amplitudes and characteristic times of fast and slow relaxation modes and the stretching exponent with calcium chloride concentration for $10 \mathrm{~g} \mathrm{~L}^{-1}$ aqueous pectin solution in the presence of $0.1 \mathrm{M} \mathrm{NaCl}$. Comparison with Fig. 9 shows that the amount of $\mathrm{CaCl}_{2}$ required to produce coupling effects similar to that observed in the absence of $\mathrm{NaCl}$ is about four times less. As observed by Axelos and co-workers $(17,27)$, the amount of calcium chloride required to obtain the sol-gel transition is larger in the absence of $\mathrm{NaCl}$. When a large amount of salt $(0.1 \mathrm{M} \mathrm{NaCl})$ is added, the ionic strength of the solution, $I$, increases. The range of the repulsive 
Coulomb interaction between the pectin molecules is decided by the Debye screening length $\kappa^{-1}$. At room temperature, $\kappa^{-1} \sim$ $3 \AA / \sqrt{I}$. Increasing the ionic strength of the solution decreases the repulsive part of the interaction energy between the pectin molecules, which would help the gelation process. Hence in the presence of $0.1 \mathrm{M} \mathrm{NaCl}$, smaller amount of $\mathrm{CaCl}_{2}$ would be sufficient to bring forth the sol-gel transition.

\section{CONCLUSIONS}

The dynamic light scattering measurements were carried out on aqueous pectin solutions, which undergo a sol-gel transition upon addition of calcium chloride. As the solution enters the gelation threshold the relaxation behavior of the correlation function is described by a stretched exponential. The stretching exponent, $\beta$, decreases rapidly as gelation progresses and the characteristic time of decay, $\tau_{\mathrm{s}}$, tends to diverge. These experimental findings are in accordance with the predictions of coupling model of Ngai and co-workers (8). The DLS measurements on pectin solutions in the presence of $0.1 \mathrm{M} \mathrm{NaCl}$ also gave similar results but the amount of calcium chloride required to induce sol-gel transition was found to be much smaller.

\section{ACKNOWLEDGMENTS}

The authors are thankful to Drs. C. Manohar and P. A. Hassan for discussions. Suggestions by Dr. Ramesh Chander on the preparation of the pectin samples are gratefully acknowledged. J.N. and V.W.D. express their gratitude to Dr. P. A. Hassan of Novel Materials and Structural Chemistry Division, Bhabha Atomic Research Centre, Mumbai, for help in DLS measurements using a BI-90 particle sizer. This work was supported by the Board of Research in Nuclear Sciences under Project No. 37/9/96-R\&D-II of the Department of Atomic Energy, Government of India.

\section{REFERENCES}

1. "Gels and Gelling Processes," Discuss. Faraday Soc. 57 (1974).

2. Adam, M., Delsanti, M., Munch, J. P., and Durand, D., Phys. Rev. Lett. 61, 706 (1988).

3. Martin, J. E., and Wilcoxon, J. P., Phys. Rev. Lett. 61, 373 (1988).
4. Martin, J. E., Wilcoxon, J. P., and Odinek, J., Phys. Rev. A 43, 858 (1991).

5. Ren, S. Z., Shi, W. F., Zhang, W. B., and Sorensen, C. M., Phys. Rev. A 45, 2416 (1992).

6. Bauer, J., and Burchard, W. J., J. Phys. II France 2, 1053 (1992).

7. Maity, S., and Bohidar, H. B., Phys. Rev. E 58, 729 (1998).

8. Ngai, K. L., Adv. Colloid Interface Sci. 64, 1 (1996).

9. Ngai, K. L., Rajagopal, A. K., and Teitler, S., J. Chem. Phys. 88, 5086 (1988).

10. Rendell, R. W., Ngai, K. L., and Mckenna, G. B., Macromolecules 20, 2250 (1987).

11. Ngai, K. L., Peng, S. L., and Tsang, K. Y., Phys. A 191, 523 (1992).

12. Ngai, K. L., and Tsang, K. Y., Phys. Rev. E 60, 4511 (1999).

13. Ngai, K. L., and Phillies, G. D. J., J. Chem. Phys. 105, 8385 (1996).

14. Thakur, B. R., Singh, R. K., and Handa, A. K., Crit. Rev. Food Sci. Nutr. 37, 47 (1997).

15. Thibault, J. F., and Rinaudo, M., Br. Polym. 17, 181 (1985); Biopolymers 25, 455 (1986).

16. Powell, D. A., Morris, E. R., Gidley, M. J., and Rees, D. A., J. Mol. Biol. 155, 517 (1982).

17. Garnier, C., Axelos, M. A. V., and Thibault, J. F., Carbohydr. Res. 240, 219 (1993).

18. Berth, G., Dautzenberg, H., and Rother, G., Carbohydr. Polym. 25, 177 (1994); 25, 187 (1994).

19. Harding, S. E., Berth, G., Ball, A., Mitchell, J. R., and de la Torre, J. G., Carbohydr. Polym. 16, 1 (1991).

20. Cros, S., Garnier, C., Axelos, M. A. V., Imberty, A., and Perez, S., Biopolymers 39, 339 (1996).

21. Axelos, M. A. V., Thibault, J. F., and Lefebvre, J., Int. J. Biol. Macromol. 11, 186 (1989).

22. Axelos, M. A. V., and Kolb, M., Phys. Rev. Lett. 64, 1457 (1990) .

23. de Gennes, P. G., "Scaling Concepts in Polymer Physics." Cornell Univ. Press, Ithaca, NY, 1979.

24. Herrmann, H. J., Derrida, B., and Vannimenus, J., Phys. Rev. B 30, 4080 (1984).

25. Derrida, B., Stauffer, D. S., Herrmann, H. J., and Vannimenus, J., J. Phys. (Paris) Lett. 44, L701 (1983).

26. Nystrom, B., Walderhaug, H., and Hansen, F. K., J. Phys. Chem. 97, 7743 (1993).

27. Axelos, M. A. V., Makromol. Chem. Macromol. Symp. 39, 323 (1990).

28. Berne, B., and Pecora, R., "Dynamic Light Scattering." Wiley, New York, 1976.

29. Ngai, K. L., and Rendell, R. W., J. Non-Cryst. Solids 131-133, 233 (1991).

30. Graessley, W. W., Polymer 21, 258 (1980). 